\title{
Inter-subject Registration of Functional and Anatomical Data Using SPM
}

\author{
P. Hellier ${ }^{1}$, J. Ashburner ${ }^{2}$, I. Corouge ${ }^{1}$, C. Barillot ${ }^{1}$, and K.J. Friston ${ }^{2}$ \\ Projet Vista, IRISA/INRIA-CNRS, Rennes, France \\ Functional Imaging Lab, Wellcome Department of Imaging Neuroscience, London, UK
}

\begin{abstract}
This paper is concerned with inter-subject registration of anatomical and functional brain data, and extends our previous work [7] on evaluation of intersubject registration methods. The paper evaluates the SPM spatial normalization method [1], which is widely used by the neuroscience community. This paper also extends the previous evaluation framework to functional MEG data. The impact of three different registration methods on the registration of somatosensory MEG data is studied. We show that the inter-subject functional variability can be reduced with inter-subject non-rigid registration methods, which is in accordance with the hypothesis that part of the inter-subject functional variability is encoded in the inter-subject anatomical variability.
\end{abstract}

Keywords: Anatomical and functional atlases, non-rigid registration, spatial normalization, MR, MEG.

\section{Introduction}

This paper is concerned with inter-subject registration of anatomical and functional brain data. Traditionally addressed with paper-based atlases, the problem of inter-subject comparison can now be tackled with electronic brain atlases [5[1014]. This has been made possible because digital images of the brain are now available, either anatomical or functional; and also thanks to the development of computers, which can now cope with enormous datasets.

Despite this progress, one has still to face the difficult problem of building such an atlas: the registration of brains of different subjects, usually achieved via registering MR brain images. Brain atlases classically rely on a template, which can also be a reference subject. Contrary to traditional paper-based atlases, electronic atlases can evolve, since new subjects can still be included in the atlas in the following way: once registered with the template, anatomical and functional data associated with the subject (i.e. segmentation maps, or functional data) can enrich the atlas characteristics (probability of an anatomical structure being present or probability of functional activations, respectively).

The quality of the atlas (in terms of accuracy and reliability) surely depends on the registration process, since the latter makes it possible to encode and decrease the anatomical inter-subject variability. Various registration methods abound in the literature, and the reader can find in [9] a comprehensive survey of these methods. Among them, SPM [1] is renowned and widespread (more than 1500 citations between 1990 and 1999 and more than 1000 SPM99 installed versions). SPM can be downloaded free of charge [3], and is a standard tool for researchers interested in neuroscience. 
This article is an extension of our previous work on validation of inter-subject brain registration [7]. In [7], we designed global and local measures to assess the registration results of 6 methods on a database of 18 subjects. The current paper evaluates the SPM99 spatial normalization method, and also extends the evaluation framework to include a validation based on dipoles localized with functional MEG.

\section{SPM Registration Method}

The SPM spatial normalization approach estimates warps by matching each skullstripped image to the skull-stripped reference. Registration involves minimizing the mean squared difference between the images, which had been previously smoothed by convolving with an isotropic $8 \mathrm{~mm}$ FWHM Gaussian kernel. The first step of each registration is to estimate a 12-parameter affine transformation, in which excessive zooms and shears are penalized by adding an additional regularization term to the cost function [2].

The next step involves nonlinear registration, which corrects for gross differences in brain shape that could not be accounted for by the affine registration alone. These warps are modeled by a linear combination of low-frequency cosine transform basis functions [1]. Displacements in each direction are parameterized by 392 basis function coefficients, making a total of 1176 parameters in total. Regularization is obtained by minimizing the membrane energy of the warps. Other than matching skull-stripped images without any voxel-specific weighting, the default settings of SPM99 [3] are employed throughout. The database is composed of 18 subjects, among which one has been chosen to be the reference. The registration has been performed by matching each subject (source volume) to the reference (target volume).

\section{Results on Anatomical Data}

In this section, the SPM registration method is evaluated according to the global and local measures presented in our previous work [7]. For each measure, we will very briefly recall its principle. The SPM results will be discussed in relation to those previously obtained from other methods [7].

In order to assess the registration process, anatomical structures have been extracted for each subject of the database. The evaluation is based upon these structures, and more precisely how they are matched after registration. Two types of measures have been designed: global and local measures. Local measures focus on the matching of cortical sulci (extracted with the method described in [8]), which are particularly relevant to study the functional organization of the brain. It should also be noted that the registration and evaluation processes are independent, leading to an objective evaluation.

\subsection{Global Measures}

Average Volume. Among the subjects of the database, one subject was chosen to be the "template", or reference subject. After registration, each subject was deformed toward the reference subject (using trilinear interpolation), and an "average" volume computed. Orthogonal sections through this average volume are presented in figure 1 . The value of 


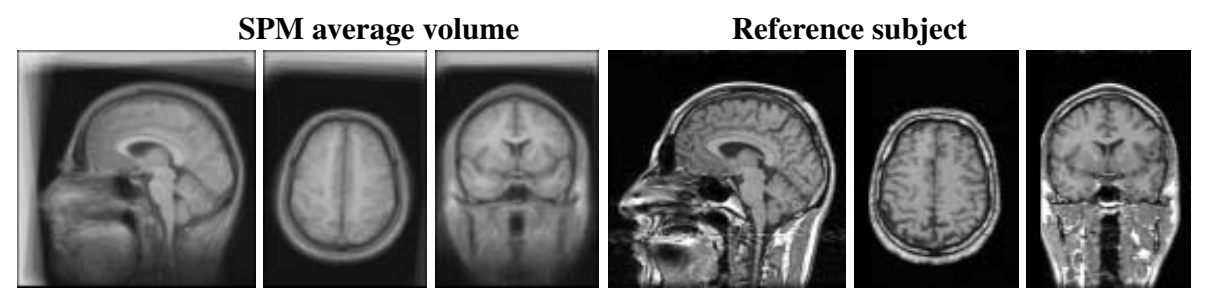

Fig. 1. Average volume for SPM registration method, to be compared with the reference subject.

Table 1. Tissue overlap and correlation of $L v v$ after registration. The mean and standard deviation of these measures through the database of subjects is computed.

\begin{tabular}{|c|c|c|}
\hline Overlap of grey matter & Overlap of white matter & Correlation of $L v v$ \\
\hline Mean - St.Dev. & Mean - St.Dev. & Mean - St.Dev. \\
\hline $94.11-0.062$ & $95.71-0.038$ & $0.246-0.0027$ \\
\hline
\end{tabular}

the mean square error between the average volume and the reference subject is 956.1 (computed only for voxels within the brain of the reference) 1 .

Tissue Overlap and Correlation of $\boldsymbol{L} \boldsymbol{v} \boldsymbol{v}$ Volumes. We designed measures based on the overlap of grey matter and white matter after registration (the total performance measure has been retained), as well as the correlation of $L v v$ volumes after registration. The $L v v$, which is related to the curvature information, has proved to be related to the cortical anatomy. The results of these two measures are presented in table 1

\subsection{Local Measures}

In addition to global measures, we designed local measures based on the matching of cortical sulci after registration. Sulci were extracted using the method described in [8], and modeled as B-spline surfaces. They are relevant landmarks for studying the functional organization of the brain.

Visualization of Deformed Sulci. We visualize in figure2 how the sulci of each subject match the corresponding sulci of the reference subject after registration. The sulci in figure 2, one per subject, should ideally match the corresponding white sulcus.

Numerical Evaluation. Beyond visualization, we can numerically assess how well sulci are matched after registration. Because sulci are defined by their control points, a distance between sulci can be defined as the distance between control points. Furthermore, we perform a Principal Component Analysis to characterize shape difference. These results are given in table 2

\footnotetext{
${ }^{1}$ This measure is not objective, in the sense that it is related to image intensities that are used to drive the registration process.
} 


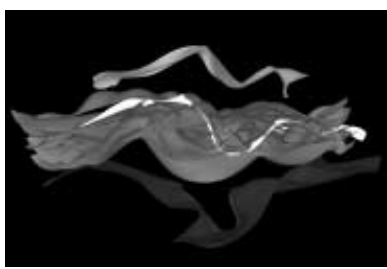

(a) central sulci

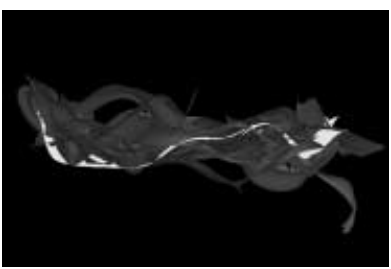

(b) superior frontal sulci

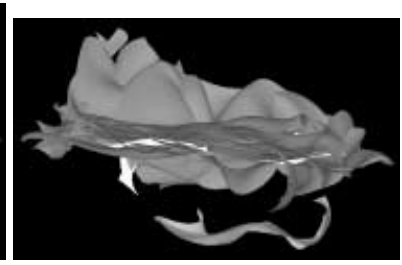

(c) lateral sulci

Fig. 2. For a given sulcus, the corresponding sulcus of each subject is deformed toward the reference subject. The corresponding reference sulcus is shown in white. Neighboring sulci ((a): postcentral sulcus and precentral sulcus. (c): superior temporal sulcus) have been also represented to illustrate the order of magnitude of the variability after registration.

Table 2. Numerical evaluation of the distance between registered and corresponding reference sulci. The first column indicates the average distance for all sulci and all subjects (the distance is expressed in voxels, the spatial resolution of the voxels being $0.93 \mathrm{~mm}$ ). The last three columns indicate the normalized trace of the covariance matrix for specific sulci: central sulci, superior frontal sulci and lateral sulci. The Principal Component Analysis provides a metric for shape differences.

\begin{tabular}{|c|c|c|c|}
\hline Mean distance & central & superior frontal & Sylvian \\
\hline 8.7 & 475 & 589 & 930 \\
\hline
\end{tabular}

\subsection{Partial Conclusion}

For global measures, the results obtained with the SPM registration method are comparable to the results obtained with methods having similar degrees of freedom [7] (that is to say, the number of estimated independent variables). Local measures have provided significantly better results, in terms of matching cortical sulci. The distance measure, as well as the shape metric, have shown that the SPM method is in average $13 \%$ better than other non-rigid registration methods.

\section{Results on Functional Data}

Spatial normalization is a crucial step for performing measurements across or between subjects. Previous work [6] has already shown that higher dimensional warping produced averaged activations with higher amplitude and more compact spatial localization. This work intends to give an insight about how much of the inter-subject functional variability can be reduced with registration methods. The underlying assumption is that the functional inter-subject variability can be decomposed into an anatomical variability (which may eventually be estimated with registration methods) and a residual functional variability.

For this study, we have chosen one of the simplest MEG activation protocols, whose anatomical localization is well-known. For each subject, the most significant dipole was retained, and deformed toward the reference subject, according to the spatial transform 
obtained by registering the MR images. We studied the variability of the dipole fog after registration. It is difficult to have an idea of the initial variability, so we chose to present results with three registration methods, that are either available on the web, or straightforward to implement:

- Method M, which is a rigid transformation by maximization of mutual information [4,15]. This method is evaluated to provide a "baseline" of the inter-subject functional variability.

- Method P, which is the Talairach and Tournoux proportional squaring system [13]. This leads to a piecewise affine transformation, defined on 12 pieces. We wil refer to this method as the T\&T registration method.

- Method S, which is the SPM registration method [1].

\subsection{Functional MEG Data}

For all methods, the functional data to register are MEG dipoles corresponding to a somatosensory activation of right hand fingers (thumb, index, little finger) performed for 15 volunteers out of the 18 subjects of our database, made up of $35+/-10$ year old healthy males, all right-handed. MEG current dipoles were reconstructed using a spatiotemporal algorithm [11] and selected by choosing the most significant one in the $45+/-15 \mathrm{~ms}$ window. Thus, three dipoles, one per finger, are available for each subject. The somatosensory paradigm chosen here is a very simple well-known one and is thus convenient to our study, since our objective is not to explain complex physiological processing but rather to study the impact of registration methods.

Despite the simplicity of the protocol, reconstruction of the sources in MEG [11] and MEG/MRI registration [12] remain challenging and generate errors. Because we aim to compare deformed dipoles with the anatomy of the reference subject (in particular sulci of the central region), we excluded dipoles that were not localized within the postcentral gyrus. It does not mean that we have eradicated reconstruction errors, but we can at least affirm that the original dipoles are correctly located. Therefore, we have kept 9 subjects for the little finger, 10 subjects for the index and 12 subjects for the thumb. As a consequence, the variability measured at the end of this process cannot be considered as an "absolute" value, but is to be trusted when comparing methods.

\subsection{Localization and Variability of Deformed Dipoles}

We first visualize where deformed dipoles are located according to the anatomy of the reference subject. In figure 3, the sulci of the central region are shown, along with the deformed dipoles.

We can numerically assess the variability of dipoles after registration, by computing the covariance matrix and its determinant, since the latter expresses the entire variation. These numerical results are presented in table 3 .

Finally, we can combine visualization and numerical results in a compact and visual way: for each group of dipoles (one per method and per finger), we compute a "mean" dipole. The dispersion of dipoles can be represented around this mean dipole. Along each axis, we compute the empirical standard deviation $\sigma$ of the dipoles coordinates. 


\section{Little finger}

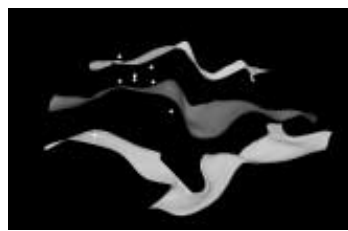

Method M

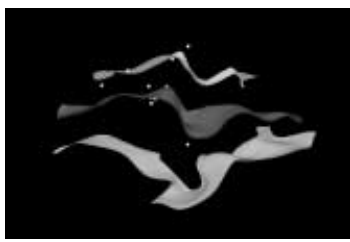

Method M

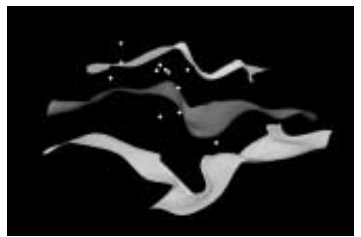

Method M

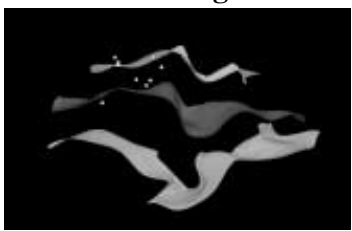

Method P

Index

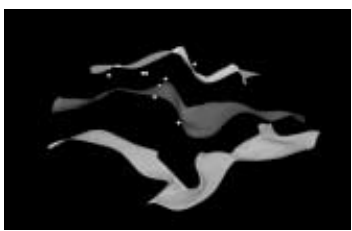

Method P

Thumb

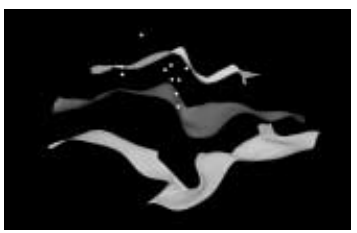

Method P

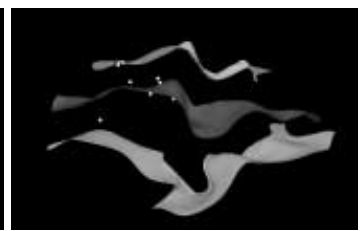

Method S

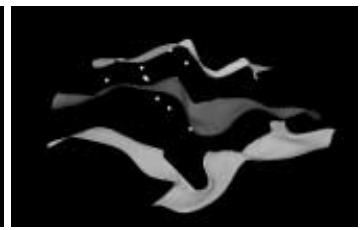

Method S

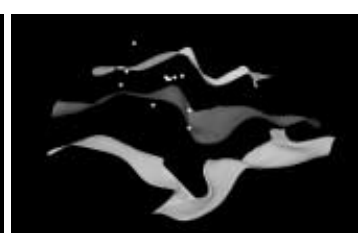

Method S

Fig. 3. For each method, and for each finger (top: little finger, middle: index; bottom: thumb) the deformed dipoles can be compared with the anatomy of the reference subject (sulci of the central region).

Table 3. Numerical results on the dispersion of dipoles. For each group of dipoles (one group per method and per finger), the determinant of the covariance matrix expresses the entire variation.

\begin{tabular}{|c|c|c|c|}
\hline & Little finger & Index & Thumb \\
\hline Method & Determinant & Determinant & Determinant \\
\hline M & 49866 & 140585 & 36782 \\
\hline P & 23561 & 52849 & 17512 \\
\hline S & 44097 & 58617 & 21216 \\
\hline
\end{tabular}

Under the assumption of a Gaussian distribution, more than $99.7 \%$ of dipoles are to be retrieved in the interval $[-3 \sigma, 3 \sigma]$. Visually, this amounts to tracing an ellipsoid centered on the mean dipole, whose radius along each axis is three times the standard deviation of the dipoles distribution on this axis. This is presented in Figure 4

\section{Conclusion}

This paper has extended our previous work [7] on the evaluation of inter-subject nonrigid registration methods. The SPM registration method [1], among the most popular 


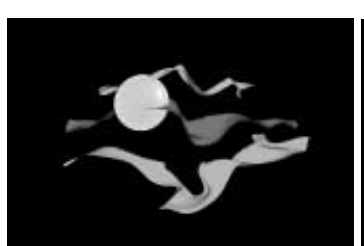

Method M

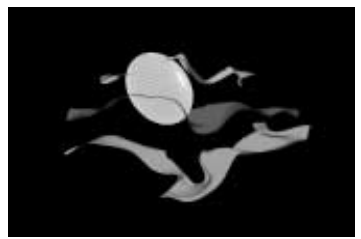

Method M

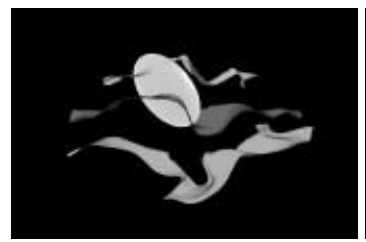

Method M

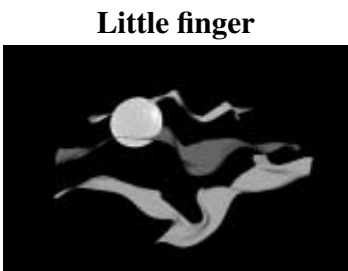

Method P

Index

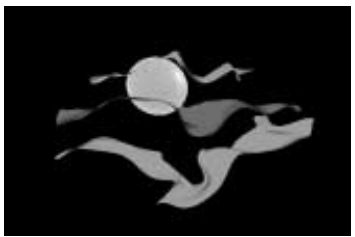

Method P

Thumb

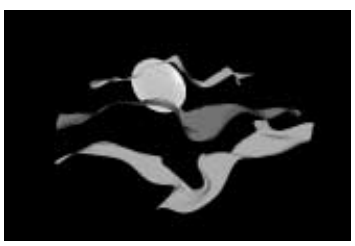

Method P

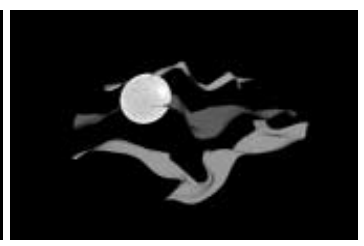

Method S

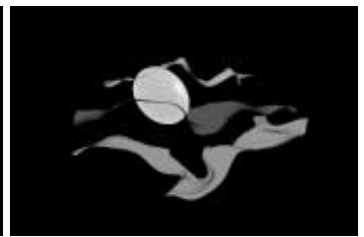

Method S

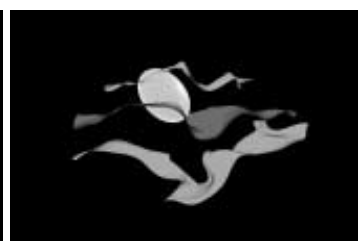

Method S

Fig. 4. For each method and each finger (top: little finger, middle: index; bottom: thumb), the variability of the deformed dipoles is represented by an ellipsoid. Under a Gaussian hypothesis, the probability of a deformed dipole being in the ellipsoid is more than 0.997 .

and widespread in the neuroscience community, has been evaluated with the global and local measures [7]. For global measures, results of the SPM registration method are in accordance with the dimension of the transformation, compared to previous evaluated methods [7]. Local measures, based on the matching of cortical sulci, show that the SPM registration method performs well, the results being significantly better than those obtained with previous methods [7].

This paper also investigates the impact of spatial normalization methods on the registration of functional data. Somatosensory MEG data are acquired and deformed toward the reference subject accordingly to the spatial registration results. The residual inter-subject variability can then be measured. In this study, a rigid registration method serves as a comparison basis for two registration methods: the T\&T proportional squaring system and the SPM registration method. The underlying assumption is that part of the inter-subject functional variability is encoded by the inter-subject anatomical variability. The study show that T\&T and SPM reduce the inter-subject variability, compared to the rigid transformation. The T\&T proportional scaling system seemed to be slightly more accurate than the SPM approach for registering functional data, at least in the central area (since the $\mathrm{T} \& \mathrm{~T}$ registration is by construction most precise in this area). 


\section{References}

1. J. Ashburner and K.J. Friston. Nonlinear spatial normalization using basis functions. Human Brain Mapping, 7(4):254-266, 1999.

2. J.Ashburner, P. Neelin, DL. Collins, A. Evans, and K.J. Friston. Incorporating prior knowledge into image registration. Neuroimage, 6:344-352, 1997.

3. http://www.fil.ion.ucl.ac.uk/spm/.

4. A. Collignon, D. Vanderneulen, P. Suetens, and G. Marchal. 3D multi-modality medical image registration using feature space clustering. In Proc. of CVRMed, pages 195-204, Nice, France, 1995.

5. A. Evans, L. Collins, and B. Milner. An MRI-based stereotaxic atlas from 250 young normal subjects. Soc. Neuroscience abstract, 18:408, 1992.

6. J.C. Gee, D.C. Alsop, and G.K. Aguirre. Effect of spatial normalization on analysis of functional data. In K. M. Hanson, editor, Proc. SPIE MI 1997: IP, SPIE (3034), pp 550-560, 1997.

7. P. Hellier, C. Barillot, I. Corouge, B. Gibaud, G. Le Goualher, D.L. Collins, A. Evans, G. Malandain, and N. Ayache. Retrospective evaluation of inter-subject brain registration. Proc. of MICCAI, LNCS number 2208, pp 258-265, 2001.

8. G. Le Goualher, C. Barillot, and Y. Bizais. Modeling cortical sulci with active ribbons. IJPRAI, 8(11):1295-1315, 1997.

9. J. Maintz and MA. Viergever. A survey of medical image registration. Medical Image Analysis, 2(1):1-36, 1998.

10. J. Mazziotta, A. Toga, A. Evans, P. Fox, and J. Lancaster. A probabilistic atlas of the human brain: theory and rationale for its development. Neuroimage, 2:89-101, 1995.

11. D. Schwartz, D. Badier, JM. Bihoué, and A. Bouliou. Evaluation with realistic sources of a new meg-eeg spatio-temporal localization approach. Brain Topography, 11(4):279-289, 1999.

12. D. Schwartz, E. Poiseau, D. Lemoine, and C. Barillot. Registration of MEG/EEG data with 3D MRI : Methodology and precision issues. Brain Topography, 9(2), 1996.

13. J. Talairach and P. Tournoux. Co-planar stereotaxic atlas of the human brain. Georg Thieme Verlag, Stuttgart, 1988.

14. P. Thompson, R. Woods, M. Mega, and A. Toga. Mathematical/computational challenges in creating deformable and probabilistic atlases of the human brain. Human brain mapping, 9:81-92, 2000.

15. P. Viola and W. Wells. Alignment by maximization of mutual information. In Proc. ICCV, pages 15-23, 1995. 